\title{
Outbreak of coagulase negative staphylococcus highly resistant to ciprofloxacin in a leukaemia unit
}

\author{
B A Oppenheim, J W Hartley, W Lee, J P Burnie
}

\begin{abstract}
Objective - To define an outbreak of bacteraemia due to coagulase negative staphylococci highly resistant to ciprofloxacin in a leukaemia unit, investigate the source and mode of spread of the outbreak strain, and assess control measures.

Design-The outbreak strain was characterised by five different typing methods. Surveillance of patients, staff, and environment was carried out during the outbreak and five months after control measures were introduced.
\end{abstract}

Setting-A unit with 10 beds for adults with leukaemia and patients receiving bone marrow transplants. The outbreak occurred during a trial of ciprofloxacin for empirical treatment of neutropenic fevers.

Interventions - Ciprofloxacin was withdrawn from use in the unit and daily bathing with chlorhexidine gluconate solution started.

Main outcome measure-The absence of bacteraemia due to the outbreak strain for five months after control measures.

Results-During the study 49 patients developed 21 episodes of bacteraemia due to the outbreak strain, which was ciprofloxacin resistant (minimum inhibitory concentration $\geqslant 128 \mathrm{mg} / \mathrm{l}$ ), susceptible to phage $155 \mathrm{~A} 9 \mathrm{C}$, and SII biotype and had characteristic immunoblot and DNA fingerprint features. There was a high amount of colonisation of patients but not staff with this strain, which was also widespread in the environment. The control measures led to rapid resolution of the outbreak and disappearance of the strain from the unit.

Conclusions-In areas where coagulase negative staphylococcal infections are common doctors must be aware of the possibility of cross infection with a single strain, and the availability of more discriminatory methods of typing will facilitate the identification and control of such episodes.

\section{Department of \\ Microbiology, Withington Hospital, Manchester M20 8LR \\ B A Oppenheim, MRCPATH, consultant microbiologist J W Hartley, MRCPATH, senior registrar}

Department of Medical Microbiology, St Bartholomew's Hospital, London

W Lee, PHD, research assistant

J P Burnie, MD, senior lecturer

Correspondence to:

Dr Oppenheim.

BrMed f 1989;299:294-7 quate. Phage typing lacks typability and reproducibility while three quarters of clinically important isolates are biotype SII. ${ }^{8}$ Antibiograms can be unreliable as the number of potential types is limited and reproducibility may be poor. ${ }^{9}$ Recently both immunoblot $^{10}$ and DNA fingerprinting ${ }^{11}$ have been applied to coagulase negative staphylococci. Immunoblot techniques depend on the pattern of protein bands and hence the phenotypic expression of antigenic determinants whereas DNA fingerprinting is determined directly by genotype. Both techniques can differentiate between isolates of coagulase negative staphylococci that are non-phage typable, identical by antibiograms, and yet clearly clinically distinct. ${ }^{11}$ We used these methods to identify an outbreak strain of coagulase negative staphylococci and subsequently examined the effect of withdrawing ciprofloxacin from the unit.

\section{Subjects and methods}

Hospital setting - Adults with leukaemia and patients receiving bone marrow transplants were housed in a unit with 10 beds consisting of six isolation rooms with filtered air under positive pressure and full reverse barrier nursing and four single rooms with conventional ventilation and standard nursing procedures. The unit had an average rate of admission of 16 a month, most being for the supervision of neutropenic episodes after chemotherapy or bone marrow transplantation. All patients had indwelling central venous catheters. Recipients of bone marrow transplants had received oral co-trimoxazole for gut decontamination. Over 18 months neutropenic patients with fever were randomised to receive either intravenous netilmicin and ciprofloxacin or netilmicin and piperacillin. Those who did not respond were treated by changing the antibiotic regimen. In patients with proved septicaemia due to coagulase negative staphylococci or infections at the site of insertion of the catheter vancomycin was added. In most cases antibiotics were continued for the duration of the neutropenia.

Epidemiological surveys-Over two weeks cultures of samples from the nose, throat, stools, urine, and site of insertion of the catheter were taken from all patients at weekly intervals. Nasal and hand cultures were performed on the five medical and 17 nursing staff. Hands were screened by both an enrichment method ${ }^{12}$ and impression of fingers on to a selective medium. Settle plates were exposed for six hours in all patients' rooms, corridors, and staff changing areas. Slit sampling was performed twice at six sites (four patient's rooms and two corridors) with a Cassela slit sampler. This survey was repeated five months after control measures were instituted, with identical procedures for patients and the environment but not including staff.

Microbiology-Blood cultures were taken from the central lines and peripheral veins of all neutropenic patients with fever before antibiotic treatment was started and during any subsequent episodes of fever. They were examined by a radiometric system (Bactec). The samples taken during the epidemiological survey were inoculated on to a selective medium $(5 \%$ blood agar containing ciprofloxacin $5 \mathrm{mg} / \mathrm{l}$ ). Plates were 
incubated for 48 hours at $37^{\circ} \mathrm{C}$. Strains isolated from this medium, which were Gram positive cocci, catalase positive, and coagulase negative, were then subcultured for further study.

Characterisation of isolates-Isolates were initially typed by the API staphylococcus system, which provided a seven digit profile for each isolate. They were subsequently phage typed and biotyped by the division of hospital infection, Colindale.

Antibiograms-were determined by a disc susceptibility method on agar (Isosensitest, Oxoid CM471) with $30 \mu \mathrm{g}$ discs of vancomycin; $10 \mu \mathrm{g}$ discs of methicillin, fusidic acid, gentamicin, and chloramphenicol; $5 \mu \mathrm{g}$ discs of erythromycin; $2 \mu \mathrm{g}$ discs of rifampicin and clindamycin; and $1 \mu \mathrm{g}$ discs of penicillin. All tests were performed at $35^{\circ} \mathrm{C}$ with the exception of that with methicillin, which was performed at $30^{\circ} \mathrm{C}$.

Immunoblot fingerprinting-was performed as described elsewhere.$^{13}$ Isolates were grown overnight

TABLE I-Details of 13 immunoblot types isolated during outbreak of bacteraemia due to coagulase negative staphylococci in leukaemia unit

\begin{tabular}{|c|c|c|c|c|c|c|c|c|c|c|c|c|c|}
\hline \multirow[b]{2}{*}{ Antigenic band (kilodalton) } & \multicolumn{13}{|c|}{ Immunoblot type } \\
\hline & 1 & 2 & 3 & 4 & 5 & 6 & 7 & 8 & 9 & 10 & 11 & 12 & 13 \\
\hline $185-190$ & + & + & + & & & + & + & + & & + & & & \\
\hline 98 & & + & & + & + & + & & + & + & & + & + & \\
\hline $\begin{array}{l}68 \\
60\end{array}$ & + & + & + & + & + & + & + & DB & DB & + & + & + & + \\
\hline 56 & & + & & & + & + & & + & + & + & & & \\
\hline 50 & + & & & & & & & + & & & & + & \\
\hline 40 & & & & & & + & & & + & & + & & + \\
\hline 37 & + & + & & + & + & + & + & + & + & + & & + & + \\
\hline
\end{tabular}

$+=$ Band present $; \mathrm{DB}=$ double band.

$\star 1=$ Outbreak type; $2-13=$ non-outbreak controls.

TABLE II-Details of 12 DNA types isolated during outbreaks of bacteraemia due to coagulase negative staphylococci in leukaemia unit

\begin{tabular}{|c|c|c|c|c|c|c|c|c|c|c|c|c|}
\hline \multirow[b]{2}{*}{ Genomic band (kilobase) } & \multicolumn{12}{|c|}{ DNA type ${ }^{\star}$} \\
\hline & A & B & $\mathrm{C}$ & $\mathrm{D}$ & $\mathrm{E}$ & $\mathrm{F}$ & G & $\mathbf{H}$ & I & J & $\mathbf{K}$ & $\mathrm{L}$ \\
\hline 9.0 & + & + & & & + & + & + & DB & & & & \\
\hline 5.5 & & DB & & & + & DB & & & & + & + & + \\
\hline $4 \cdot 4$ & + & & + & & & + & & & & + & & + \\
\hline $4 \cdot 0$ & & & & & & & & + & & & & \\
\hline 3.6 & + & & & & + & & + & & & + & + & + \\
\hline 2.9 & + & & + & & + & & + & & + & + & & + \\
\hline $2 \cdot 3$ & & & + & + & & + & $\mathrm{DB}$ & & & & & \\
\hline 1.9 & DB & & DB & & & DB & $\mathrm{DB}$ & & & DB & & DB \\
\hline 1.7 & & & + & & DB & DB & + & & & $\mathrm{DB}$ & + & $\mathrm{DB}$ \\
\hline
\end{tabular}

$+=$ Band present $; \mathrm{DB}=$ double band.

$\star \mathrm{A}=$ Outbreak type; $\mathrm{B}-\mathrm{L}=$ non-outbreak controls.

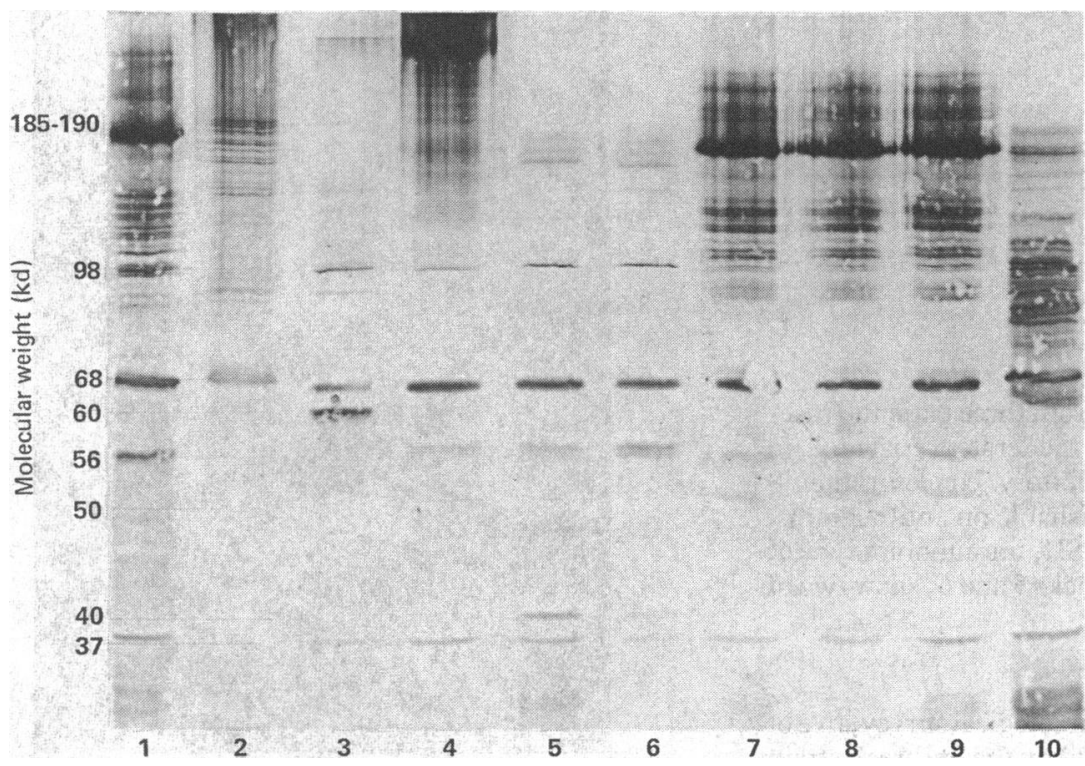

FIG 1-Immunoblot types 1-7. Track 1 shows type 1 (case 1); track 2, type 3 (case 2); track 3, type 4 (case 3); track 4, type 5 (case 4); tracks 5 and 6, type 6 (cases 22 and 23); tracks 7-9, type 1 (outbreak strain); and track 10, type 7 (case 14)

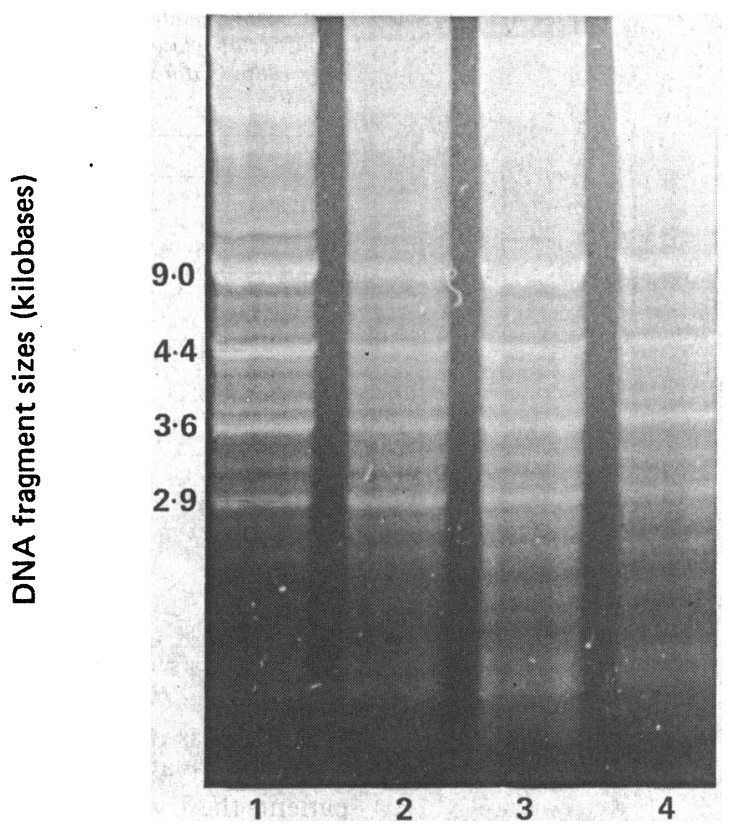

FIG 2-DNA fingerprints of four outbreak isolates

in soya broth (Tryptone) and the pellet was disrupted by incubation with lysostaphin at $37^{\circ} \mathrm{C}$ for 30 minutes. The soluble supernatant obtained was run on a $10 \%$ sodium diodecyl sulphate-polyacrylamide gel and transferred on to a nitrocellulose membrane. This was then stained by a rabbit hyperimmune antiserum raised against one of the isolates from the outbreak.

DNA fingerprinting - was performed as detailed elsewhere." Isolates were disrupted by lysostaphin degradation, and after phenol-chloroform extraction the DNA was digested by the restriction enzyme $E c o R I$. This digestion was carried out to completion at $37^{\circ} \mathrm{C}$ for two hours. The digests were run on a horizontal gel containing $0 \cdot 8 \%$ agarose with a TRISborate-EDTA buffer system. After electrophoresis the gel was visualised under ultraviolet light and photographed with Polaroid film (type 57).

Control measures-Ciprofloxacin was withdrawn from use in the unit, and the combination of netilmicin and piperacillin became the first line antibiotic treatment. Daily whole body bathing with chlorhexidine gluconate solution (Hibiscrub, Imperial Chemical Industries) was started. Staff were alerted to the presence of the outbreak strain and asked to pay special attention to infection control procedures.

\section{Results}

\section{PATIENT ISOLATES}

All isolates of coagulase negative staphylococci resistant to ciprofloxacin obtained during severe bacteraemias in patients in the leukaemia unit were typed by all five techniques. This showed the presence of an outbreak strain susceptible to phages 155 and A9C; resistant to penicillin, methicillin, erythromycin, clindamycin, and gentamicin; sensitive to chloramphenicol, rifampicin, fusidic acid, and vancomycin; of SII biotype (API staphylococcus profile 6704112); and resistant to ciprofloxacin (minimum inhibitory concentration $\geqslant 128 \mathrm{mg} / 1$ ). We designated this outbreak strain as immunoblot type 1 (table I) and DNA fingerprint type A (table II). Figures 1 (tracks 7, 8, and 9) and 2 (tracks 1-4) show examples of outbreak isolates.

The first two bacteraemias due to this outbreak strain occurred seven months after ciprofloxacin was started in two patients who had had prolonged periods of neutropenia, and bacteraemias due to these isolates continued throughout the next 12 months (table III). 
TABLE III - Number of patients with bacteraemias due to coagulase negative staphylococci after treatment with ciprofloxacin introduced in May 1986. Outbreak strain was first isolated in November 1986 and control measures were introduced in November 1987

\begin{tabular}{|c|c|c|}
\hline & Outbreak strain & Non-outbreak strain \\
\hline Oct 1986 & & 2 \\
\hline Nov & 2 & 4 \\
\hline Dec & & 2 \\
\hline Jan 1987 & 1 & 3 \\
\hline Feb & 2 & 2 \\
\hline Mar & 1 & 4 \\
\hline Apr & 1 & 4 \\
\hline May & 3 & 2 \\
\hline Jun & 1 & 1 \\
\hline Jul & 2 & 4 \\
\hline Aug & 1 & 2 \\
\hline Sep & 1 & 2 \\
\hline $\mathrm{Oct}^{\star}$ & 4 & \\
\hline Nov & 1 & 1 \\
\hline Dec & 1 & 3 \\
\hline Jan 1988 & & 1 \\
\hline Feb & & 2 \\
\hline Mar* & & 3 \\
\hline
\end{tabular}

Bacteraemias due to coagulase negative staphylococci occurred in 49 patients during that period. In 19 patients these were due to the outbreak strain. Of these 19 patients, five had two separate bacteraemic episodes, one had three, and one had four. Only one episode occurred after control measures were started. As controls, 21 cases of septicaemia due to coagulase negative staphylococci sensitive to ciprofloxacin (minimum inhibitory concentration $<1 \mathrm{mg} / \mathrm{l}$ ) (cases 1-21) were examined. Typing showed most to be nonphage typable and biotype SII (table IV). Immunoblot fingerprinting produced 12 separate types as defined by a difference in at least three antigenic bands. ${ }^{10}$ Figure 1 shows isolates from cases $1,3,4,12$, and 14 . DNA fingerprinting showed 11 separate types as defined by a difference in at least one band. ${ }^{11}$

TABLE IV-Details of ciprofloxacin sensitive stains isolated from blood cultures during outbreak of bacteraemia due to coagulase negative staphylococci in leukaemia unit

\begin{tabular}{|c|c|c|c|c|}
\hline Case No & Phage type & Biotype & Immunoblot type & DNA type \\
\hline 1 & $\ddagger$ & SII & 2 & G \\
\hline 2 & 155/A9C & SII & 5 & $\mathrm{~F}$ \\
\hline 3 & $\ddagger$ & SII & 4 & B \\
\hline 4 & $\neq$ & SII & 5 & $\mathrm{~F}$ \\
\hline 5 & $\stackrel{+}{\ddagger}$ & SII & 10 & J \\
\hline 6 & $456 / 165$ & SII & 3 & B \\
\hline 7 & $\ddagger$ & SIV & 11 & $\mathrm{~F}$ \\
\hline 8 & $\neq$ & SII & 12 & G \\
\hline 9 & $\ddagger$ & SII & 4 & H \\
\hline 10 & $\underset{+}{\ddagger}$ & SII & 11 & $\mathrm{~K}$ \\
\hline 11 & $\ddagger$ & SII & 5 & $\mathrm{D}$ \\
\hline 12 & $\ddagger$ & SVI & 3 & B \\
\hline 13 & $\ddagger$ & SVI & 4 & $\mathrm{E}$ \\
\hline 14 & $\ddagger$ & SII & 7 & $\mathrm{C}$ \\
\hline 15 & $\ddagger$ & SVI & 13 & $\mathbf{H}$ \\
\hline 16 & $27 / 48$ & SII & 9 & G \\
\hline 17 & $27 / 37 / 155$ & SII & 4 & J \\
\hline 18 & $\ddagger$ & SII & 8 & $\mathrm{E}$ \\
\hline 19 & $275 / 82 / \mathrm{B} 1$ & SII & 7 & $\mathrm{~F}$ \\
\hline 20 & $\neq$ & SVI & 3 & $\mathrm{~F}$ \\
\hline 21 & $\ddagger$ & SII & 7 & I \\
\hline
\end{tabular}

Isolates were also available from three patients (cases 22-24) in whom there was moderate resistance to ciprofloxacin (minimum inhibitory concentration 8 $\mathrm{mg} / \mathrm{l}$ ). These were indistinguishable on antibiograms, untypable by phages, biotype SII, immunoblot type 6 , and DNA type L. Figure 1, tracks 5 and 6, show two of the immunoblots.

\section{EPIDEMIOLOGICAL RESULTS}

At the time of the first epidemiological survey, five of the 10 inpatients were colonised by the outbreak strain (three in faeces, two nasally) and three of these subsequently developed bacteraemia. Coagulase nega- tive staphylococci resistant to ciprofloxacin were not found in any specimens taken from staff. Highly resistant strains with an API staphylococcus profile 6704112 were isolated from 36 of 44 settle plates (maximum counts 140 colony forming units $/ \mathrm{m}^{3}$ air) and at four of the six sites tested on the first occasion and five of the six sites tested on the second occasion by slit sampling (maximum counts 6 colony forming units $/ \mathrm{m}^{3}$ air). Twenty of these isolates were examined, and 18 were of the outbreak type according to the five typing methods. These came from five of the six isolation rooms, three of the four single rooms, the corridor, the staff changing room, and a patient's bathroom and toilet in the non-isolation area.

\section{EFFECTS OF CONTROL MEASURES}

The control measures led to the rapid resolution of the outbreak. Isolates from the first seven septicaemias due to coagulase negative staphylococci that occurred after the institution of control measures were typed. One was the outbreak strain in a patient who had previously been colonised with it. The remaining six were biotype SII, non-phage typable, and had an antibiogram distinct from that of the outbreak strain. They were sensitive to ciprofloxacin and produced three separate immunoblot types (table V). All isolates

TABLE $\mathrm{v}-$ Details of coagulase negative staphylococci from blood cultures after stopping treatment with ciprofloxacin

Case No Phage type Biotype Antibiogram Immunoblot type ${ }^{\star}$ DNA type

\begin{tabular}{llllrl}
25 & $\ddagger$ & SII & $\xi$ & 7 & $\mathrm{C}$ \\
26 & $\ddagger$ & SII & $\xi$ & 3 & $\mathrm{D}$ \\
27 & $\ddagger$ & SII & $\xi$ & 3 & $\mathrm{E}$ \\
28 & $\ddagger$ & SII & $\xi$ & 11 & F \\
29 & $\ddagger$ & SII & $\xi$ & 7 & B \\
30 & $\ddagger$ & SII & $\xi$ & 3 & H \\
\hline
\end{tabular}

$\star 2-13=$ Non-outbreak control.

$+B-L=$ Non-outbreak control.

$\ddagger$ Not phage typable.

ENon-outbreak antibiogram.

produced their own unique DNA fingerprints, and figure 3 illustrates five of these. We were unable to isolate the outbreak strain from any patients or sites tested in the repeat survey performed five months after the control measures were started.

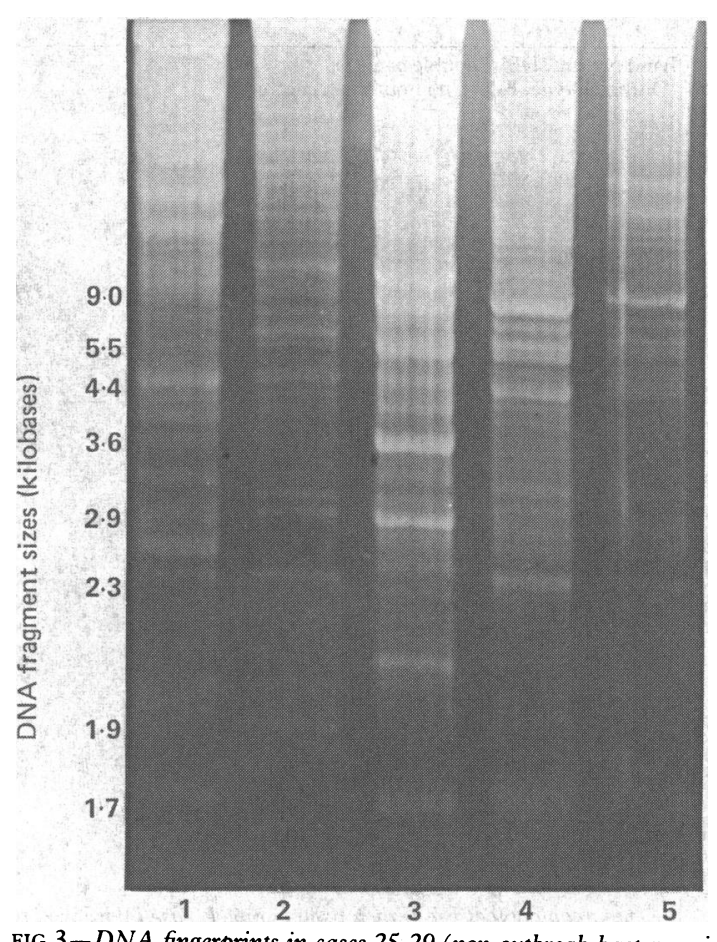

FIG 3-DNA fingerprints in cases 25-29 (non-outbreak bacteraemic strains isolated after control methods started), tracks $1-5$ respectively 


\section{Discussion}

Outbreaks in which coagulase negative staphylococci have been implicated are not common. Van den Broek et al reported an epidemic of prosthetic valve endocarditis in which a surgeon was found to be the source of contamination. ${ }^{14}$ In an outbreak described by Houang et al staff in an intensive care unit were implicated in the spread of the infecting strain. ${ }^{15}$ We have reported probably the first example of a coagulase negative staphylococcus highly resistant to ciprofloxacin causing an outbreak of septicaemia in a leukaemia unit where the airborne route seemed to be the major mode of spread. The identification of this outbreak was facilitated by the availability of new typing methods, without which it would have been impossible to be confident that the ciprofloxacin resistant isolates did not constitute several different strains, each one being resistant because of a separate mutation.

Typing systems can be judged by three criteria: typability, reproducibility, and discrimination. All isolates were fingerprinted with both immunoblot and DNA typing systems, and reproducibility was excellent provided that the conditions were standardised. The degree of discrimination with immunoblot fingerprinting has been shown for biotype SII isolates to be greater than that with DNA fingerprinting. ${ }^{11}$ In the current study the 30 non-outbreak isolates produced 13 immunoblot types and 12 DNA types. The outbreak strain was susceptible to phages 155 and A9C, and this distinguished it from all except one (case 2) of the control isolates, only five of which were phage typable. Biotyping failed to distinguish the outbreak strain from most non-outbreak isolates (table V). Phage typing and biotyping also missed a small episode of cross infection due to a strain that was moderately resistant to ciprofloxacin (minimum inhibitory concentration $8 \mathrm{mg} / 1$ ). This strain had its own unique DNA type (L) and immunoblot fingerprint (type 6).

The results of the epidemiological survey confirmed that the outbreak strain could survive in the environment and that staff were not an important vector of transmission. New patients probably became colonised by the strain while receiving prolonged treatment with broad spectrum antibiotics. Ciprofloxacin has a distinct effect on reducing gut flora ${ }^{16}$ and may also affect commensal flora at other sites, allowing highly resistant organisms to occupy these niches.

The strain was highly resistant to ciprofloxacin (minimum inhibitory concentration $\geqslant 128 \mathrm{mg} / 1$ ). This is rare as previous ciprofloxacin resistant isolates of staphylococci have had minimum inhibitory concentrations not greater than $8 \mathrm{mg} / 1 .{ }^{56}$ Recently resistance to ciprofloxacin in response to prolonged treatment has been reported in the methicillin resistant Staphylococcus aureus. ${ }^{17}{ }^{18}$ In the case of Gram negative bacteria resistance has been due to chromosomal mutation rather than plasmid transmission..$^{190}$ This is important as withdrawal of the compound should lead to the disappearance of resistance. There should be no pool of plasmid mediated ciprofloxacin resistant bacteria from which resistance may rapidly emerge if the drug is reintroduced.

When ciprofloxacin was removed from the unit the outbreak rapidly ended. There was no evidence for a residual group of ciprofloxacin resistant isolates of coagulase negative staphylococci, suggesting that the emergence of the resistance was due to chromosomal mutation rather than plasmid transfer. The rapidity with which these strains appeared is a limitation on the widespread prescription of ciprofloxacin for life threatening illnesses. Their control by the simple removal of the drug is unusual and encouraging.

In summary, we have shown that outbreaks of coagulase negative staphylococci leading to septicaemia may occur among neutropenic patients and that sepsis of the central catheter is not necessarily due to the patient's own strain. In units with a high incidence of infection with coagulase negative staphylococci we suggest that isolates should be phage typed and biotyped and an antibiogram obtained. If these techniques lack discrimination then DNA or immunoblot fingerprinting should be considered. This enables cross infection by a more virulent strain to be identified and controlled, in this case by stopping the widespread use of ciprofloxacin.

We thank Drs J H Scarffe and G Morgenstern for permission to report this outbreak and Miss P Tobin, nursing officer (infection control), for help with the epidemiological surveys.

I Wade JC, Schimpff SC, Newman KA, Wiernik PH. Staphylococcus epidermidis: an increasing cause of infection in patients with granulocytopenia. Ann Intern Med 1982;97:503-8.

2 Bender JW, Hughes WT. Fatal Staphylococcus epidermidis sepsis following bone marrow transplantation. Johns Hopkins Medical fournal 1980;146: $13-5$.

3 Winston DJ, Dudnick DV, Chapin M, Ho WG, Gale RP, Martin WJ. Coagulase-negative staphylococcal bacteraemia in patients receiving immunosuppressive therapy. Arch Intern Med 1983;143:32-6.

4 Pfaller MA, Herwaldt LA. Laboratory, clinical and epidemiological aspects of coagulase-negative staphylococci. Clinical Microbiology Reviews 1988; 1:281-99.

5 Humphreys H, Mulvihill E. Ciprofloxacin resistant Staphylococcus aureus. L.ancet 1985 ;ii: 383 .

6 Smith GM, Cashmore C, Leyland MJ. Ciprofloxacin resistant staphylococci. Lancet 1985;ii:949.

7 de Saxe MJ, Crees-Morris JA, Marples R, Richardson JF. Evaluation of current phage-typing systems for coagulase negative staphylococci. Zentral blatt für Bacteriologie 1981;10(suppl): 197-204

8 Marples RR. Laboratory assessment in the epidemiology of infections caused by coagulase negative staphylococci. F Med Microbiol 1986;22:285-6.

9 Christensen GD, Parisi JT, Bisno AL, Simpson WA, Beachey EH. Characterization of clinically significant strains of coagulase negative staphylococci. f Clin Microbiol 1983;18:258-9.

10 Burnie JP, Lee W, Matthews RC, Bayston R. Immunoblot fingerprinting of coagulase negative staphylococci. I Clin Pathol 1988;41:103-7.

11 Burnie JP, Lee W. A comparison of DNA and immunoblot fingerprinting of the S11 biotype of coagulase negative staphylococci. Epidemiol Infect 1988;101:203-12.

12 Casewell MW, Phillips I. Hands as route of transmission for Klebsiella species. BrMed F 1977;ii:1315-7.

13 Burnie JP, Matthews RC. Immunoblot analysis: a new method for fingerprinting hospital pathogens. F Immunol Methods 1987;100:141-6.

14 Van den Broek PJ, Lampe AS, Berbee GAM, Thompson J, Mouton RP. Epidemic of prosthetic valve endocarditis caused by Staphylococcus epidermidis. Br Med J 1985;291:949-50.

15 Houang ET, Marples RR, Weir I, Mourant AJ, de Saxe MJ, Singleton B. Problems in the investigation of an apparent outbreak of coagulase-negative staphylococcal septicaemia following cardiac surgery. I Hosp Infect 1986; 8:224-32.

16 Rozenberg-Arska M, Dekker AW, Verhoef J. Ciprofloxacin for selective decontamination of the alimentary tract in patients with acute leukaemia during remission induction treatment; the effect on faecal flora. $\mathcal{F}$ Infect Dis 1985;152:104-7.

17 Isaacs RD, Kunke PJ, Cohen RL, Smith JW. Ciprofloxacin resistance in epidemic methicillin resistant Staphylococcus aureus. Lancet 1988;ii:843.

18 Milne LM, Faiers MC. Ciprofloxacin resistance in epidemic methicillin resistant Staphylococcus aureus. Lancet 1988;ii:843.

resistant Staphylococcus aureus. Lancet 1988;ii:843.
19 Wolfson JS, Hooper DC. The fluoroquinolones: structures, mechanism of action and resistance, and spectra of activity in vitro. Antimicrob Agents action and resistance, and

20 Hooper DC, Wolfson JS, Ng EY, Swartz MN. Mechanisms of action and resistance to ciprofloxacin. Am F Med 1987;82(suppl 4A):12-21.

(Accepted 18 May 1989)

\section{ONE HUNDRED YEARS AGO}

An endeavour is being made to induce the Council of the Girls' Public School Company to introduce a systematic course of lectures on Physiology and Hygiene into their schools. Some of the ladies of the Council appear to fear that the selection of subjects might be difficult. Dr. Schofield, one of the lecturers of the National Health Society, attended a recent meeting and gave explanations which may, it is hoped, remove these difficulties. It is not a little strange that in the course of the modern education of women, the most essential of all knowledge should be withheld - that, namely, of the elementary facts of physiology and the laws of health, as to which correct elementary ideas are essential, not only to their own well-being but to that of the families of which they are ultimately to be the mistresses. (British Medical Fournal 1889; ii:25) 